\title{
A digital thumbs-up for PACS \& RIS
}

Going digital has been one of the great experiences of my professional life in radiology. A properly functioning picture archiving and communications system (PACS) has brought new interaction between the radiology department and our clinical colleagues. The centralisation and control of the department make it a pleasure to advise and train registrars. All the images are stored and available for comparison, and it is possible to alert certain departments on radiation control. The other day I made a call on behalf of a 16-day-old infant who had been irradiated 28 times during his short lifetime.

'The future's so bright, you gotta wear shades.' (with acknowledgement to Timbuk3); but only if we plan correctly. We need to look at interactive systems within and outside our national borders. Hence the request to Otto Schulze to introduce the first African PACS and RIS Edu-conference, supported by the Radiological Society of South Africa. Now is the time to lay the solid foundation of an integrated continental system. The next generation will thank us for that.

\section{Jan Lotz}

Editor-in-chief

\section{First African PACS \& RIS Edu-Conference: A practical approach to PACS \& RIS within Africa}

Picture archiving and communications systems and radiology information systems (PACS \& RIS) is not a new technology, either internationally or in South Africa. Most private practices as well as academic training institutions have some form of digitising imaging equipment in operation. Unfortunately, not all institutions are obtaining the same results from their PACS \& RIS installations; and this is the issue that will be addressed in the Edu-Conference.

The focus of the conference is not to set out why one should purchase a PACS \& RIS or which system to buy - the PACS \& RIS vendors are best suited to do this and to explain their benefits and special features. The purpose of the conference is to provide guidance on how to purchase, implement and maintain a PACS \& RIS to ensure that you will receive the benefits that are promised in the brochures.

Purchasing a PACS \& RIS is not as simple as purchasing a modality; it is not a modality but an information system requiring integration into the business processes of the institution. Although patient administrative information systems are widespread across South Africa, their use has remained administrative, and very few have made the transition to the clinical environment. Moving into the clinical environment is the distinguishing factor of PACS \& RIS. For the first time, we need to define clinical business processes and start regarding ourselves as elements in a factory making a product - the product being patient care, with the variables of quality of patient care and speed of patient care. By comparison, the implementation in a factory of, for example, an SAP system (an information system that manages and facilitates business processes, which is comparable with RIS at a basic level) requires considerable resources for successful implementation, maintenance and optimisation. The differences in the benefits derived from the installation depend on the resources allocated to the process; this is why some practices realise the benefits from PACS \& RIS as promised in the vendor's glossy brochures, whilst others regard it as a financial and productivity burden.

Successful implementation requires the active involvement of all members of the practice during implementation, and also their ongoing involvement in workflow evaluation, identifying bottlenecks and optimising the workflow. Furthermore, the system has to be maintained, and the errors that invariably arise must be attended to before they have disruptive consequences. The job of the PACS \& RIS administrator, which is a new position within the radiology environment, is to attend to the database management and guide workflow optimisation. Information and communications technology (ICT) support of sufficient capacity and knowledge is crucial to supporting the clinical IT environment of 24/7 service. Effective change management procedures must be in place to ensure that upgrades do not cause service delivery problems. Agreement on, and managing of, the service level agreement with the vendor is the final touch that can make the solution fly or fail.

All these issues will be discussed during the Edu-Conference, with emphasis on examples from the African environment. The programme includes: case presentations from two practices in South Africa, focusing on the lessons learned during implementation; a walk-about through Tygerberg Hospital to demonstrate certain concepts; a demonstration of the concepts, with reference to integration and working within a low bandwidth environment; and an open-panel discussion to address questions that delegates experience in their own practices.

The nearly 300 delegates cover a wide spectrum - radiologists, clinicians, radiographers, clinical engineers, hospital managers and several vendors of not only PACS \& RIS products but also ICT support and solutions - and come from across the country as well as other African states. Networking opportunities with colleagues and vendors alike will abound during tea breaks and lunches, the welcoming function and social dinner. Vendors will display their products in the exhibition hall.

By the end of the Edu-Conference, three questions are intended to have been resolved: ( $i$ ) Is there a need to create a body to serve as a forum for individuals involved with PACS \& RIS, for gathering and sharing information and advice? (ii) Is there a need for creating a PACS \& RIS administrator course? and (iii) Is there a need to hold more such information sessions, and in what format?

\section{Otto Schulze}

